\title{
Percepção de Risco e Comportamentos de Saúde em Relação ao Sexo Casual em Universitárias
}

\author{
Andresa Pinho Soster ${ }^{1}$ \\ Miguel Luis Alves de Soura ${ }^{1}$ \\ Elisa Kern de Castro ${ }^{1}$ \\ ${ }^{1}$ Universidade do Vale do Rio dos Sinos (Unisinos), São Leopoldo, RS, Brasil
}

\begin{abstract}
Resumo
Este estudo buscou explorar e comparar a percepção dos riscos à saúde física e os comportamentos de saúde do sexo casual entre universitárias com (CEX) e sem experiência (SEX) de sexo casual. Participaram 1.133 universitárias brasileiras (média de idade igual a 21,05 anos, $D P=2,05)$, a maioria nascidas e residentes na região Sul do país, que responderam a um questionário on-line com questões sobre a percepção dos riscos físicos, comportamentos de saúde, comportamento e histórico sexual. Foi encontrada diferença significativa nos comportamentos de saúde e nas percepções de risco entre os grupos. O grupo CEX apresentou mais comportamentos de saúde e cuidados que o grupo SEX. Embora o sexo casual esteja relacionado aos comportamentos de risco, neste estudo, as mulheres CEX apresentaram mais medidas de proteção à saúde que as mulheres SEX. Palavras-chave: comportamento psicossexual, atitudes sexuais, mulheres - higiene e saúde, psicologia da saúde, estudantes universitários
\end{abstract}

Risk Perception and Health Behaviors in relation to Casual Sex in College Women

\begin{abstract}
This study aimed to explore and compare the perceptions of physical health risks and healthy sex behaviors among female university students with (CEX) and without (SEX) experience in casual sex. With a comparative cross-sectional design, the study included 1,133 Brazilian female university students (mean age 21.05 years, $\mathrm{SD}=2.05$ ), mostly born and resident in the southern region of the country, who completed an online questionnaire about their perception of physical risks, their health behaviors, and their sexual behavior and history. There was a significant difference in health behaviors and risk perceptions between the two groups, where the CEX group showed higher levels of healthy behaviors and care than the SEX group. Although casual sex is related to risk behaviors, in this study, women in the CEX group reported taking more health protection measures than those in the SEX group.

Keywords: psychosexual behavior; sexual attitudes; women - hygiene and health; health psychology; college students.
\end{abstract}

Percepción de Riesgo y Conductas de Salud en relación al Sexo Casual en Estudiantes Universitárias

\section{Resumen}

La investigación tuvo como objetivo explorar y comparar la percepción de riesgo con la salud física y las conductas de salud relacionadas al sexo casual entre estudiantes universitarias con (CEX) y sin experiencia (SEX) de sexo casual. Participaron 1.133 estudiantes universitarias brasileñas (edad media de 21,05 años, DS=2,054), mayoritariamente nacidas y residentes de la región sur del país, que contestaron a un cuestionario online con preguntas sobre percepción de riesgos físicos, sus conductas de salud e historial sexual. Se pudo observar una diferencia significativa en los comportamientos de salud y en las percepciones de riesgo entre los grupos. El grupo CEX presentó más conductas saludables y de cuidados que el grupo SEX. Aunque el sexo casual esté relacionado con conductas de riesgo, en este estudio las mujeres CEX presentaron más medidas de protección a la salud que las mujeres del grupo SEX.

Palabras clave: conducta psicosexual; actitudes sexuales; mujeres: higiene y salud; psicología de la salud; estudiantes universitarios.

\section{Introdução}

O sexo casual é um tipo de experiência na qual ocorrem encontros sexuais entre pessoas que não se conhecem, ou se conhecem pouco, e têm sido um tipo de experiência comum entre jovens universitários ao redor do mundo (Bersamin et al., 2014; Claxton \& van Dulmen, 2013; Fielder, Walsh, Carey, \& Carey, 2014; Vrangalova, 2015). Comumente, trata-se de um encontro sem planejamento prévio, onde os envolvidos não tem o objetivo de construir uma relação duradoura (Campbell, 2008; Wentland \& Reissing, 2014, 2011). Grande parte do público universitário encontra-se na fase da adultez emergente, um período do desenvolvimento situado na faixa dos 18 aos 25 anos. A literatura aponta que é justamente nesse período que os jovens costumam dar início ao engajamento em experiências sexuais diversas, com o objetivo de explorar sua identidade e sexualidade (Arnett, 2000; Claxton \& van Dulmen, 2013). 
O sexo fora de relacionamentos formais já foi referido por alguns estudos como um fator de risco à saúde sexual e bem-estar (Bersamin et al., 2014; Fielder \& Carey, 2010; Hoyle, Fejfar, \& Miller, 2000; Lyons, 2016). Além disso, um estudo recente apontou que o perfil de jovens na fase da adultez emergente, com potencial risco para o contágio de ISTs (infecções sexualmente transmissíveis), seriam os que tinham em seu histórico: três ou mais parceiros(as) casuais, início da vida sexual antes dos 18 anos, e relações sexuais com pessoa que conheceram no mesmo dia (Lyons, 2016). No entanto, a literatura aponta que o número de pessoas infectadas por ISTs têm crescido exponencialmente dentro de relações estáveis devido à redução do uso consistente do preservativo (Dourado, MacCarthy, Reddy, Calazans, \& Gruskin, 2015). Uma investigação sobre o comportamento sexual da população brasileira apontou que a frequência de utilização de preservativos é maior entre pessoas que possuem parceiros eventuais $(78,6 \%)$, e menor entre as pessoas que vivem relações estáveis (33,1\%) (Berquó, Barbosa, \& Lima, 2008). Outros dados importantes advêm de uma revisão narrativa que investigou fatores associados ao uso do preservativo por brasileiros que envolviam diferentes grupos populacionais (heterossexuais, bissexuais, homossexuais, adolescentes, profissionais do sexo) (Dourado et al., 2015). Ao comparar o uso de preservativo com parceiros sexuais estáveis e casuais, todos os estudos revisados demonstraram maior frequência no uso de preservativo entre parceiros sexuais casuais (Dourado et al., 2015). Na medida em que a incidência do contágio por ISTs cresce em relações formais, assim como a frequência de não utilização do preservativo, torna-se de extrema relevância investigar demais riscos associados à prática de sexo casual.

A literatura especializada aponta que o sexo casual apresenta relação com riscos e consequência negativas para a saúde, principalmente para a saúde da mulher (Claxton \& van Dulmen, 2013; Fielder et al., 2014; Hoyle et al., 2000; Mendão \& Biscaia, 2015). Dessa forma, a prática de sexo casual estaria associada à diminuição do risco percebido para a saúde (Abbey, Saenz, \& Buck, 2005; Mendão \& Biscaia, 2015). Esse fato torna-se um problema, na medida em que a diminuição da percepção de risco associada à prática de sexo casual pode contribuir com a não adesão à condutas adequadas, como a utilização do preservativo, contribuindo inclusive com a contaminação por ISTs (Claxton \& van Dulmen, 2013; Fielder et al., 2014; Gilchrist, Smith, Magee, \& Jone, 2012; Mendão \& Biscaia, 2015).
Independentemente do tipo de relação, a falta de adesão ao uso de preservativo nas relações sexuais oferece consequências negativas e impactos na saúde ginecológica, sexual e reprodutiva da mulher (Lyons, 2016; Plummer et al., 2016). ISTs, como o HPV, podem comprometer a saúde das mulheres, por meio do desenvolvimento de infecções, lesões uterinas e câncer de colo do útero (Plummer et al., 2016). Outras doenças, como clamídia, gonorreia, sífilis, HPV, herpes genital, hepatite $\mathrm{B}$, podem causar doenças e danos à sua saúde reprodutiva, além de prejuízos psicológicos, como depressão e baixa autoestima (Bersamin et al., 2014; Fielder \& Carey, 2010; Organização Mundial da Saúde, 2013). Além disto, estudos recentes têm associado a incidência de outros tipos de câncer relacionado à hepatite B (câncer de fígado) e ao HPV (colo do útero, vulva, vagina, laringe, faringe, boca, ânus (Petito, Oliveira Júnior, Petito, \& Saddi, 2015; Plummer et al., 2016).

A exposição aos riscos em saúde está relacionada ao conceito de percepção de risco (Leventhal, Phillips, $\&$ Burns, 2016). Esse conceito pode ser compreendido como uma avaliação individual que as pessoas apresentam acerca dos seus próprios riscos, envolvendo crenças e valores que dão significado a eles (Slovic, 2000). Nesse sentido, o risco real frente a um problema de saúde pode diferir do risco percebido pelo indivíduo (Leventhal et al., 2016). No caso do uso de preservativo, ele pode ser utilizado apenas em situações que são percebidas com maior risco pelos indivíduos (Dourado et al., 2015). Um estudo sobre conhecimento, atitude e prática do uso de preservativo em universitários brasileiros demonstrou que a percepção de confiança nas relações formais foi a principal justificativa para a não utilização do preservativo (Alves \& Lopes, 2008). A confiança no parceiro sexual pode levar a mulher a diminuir a percepção de risco e vulnerabilidade e, consequentemente, promover o desuso do preservativo (Harvey et al., 2007).

Dados recentes apontam que as taxas de incidência do contágio por ISTs em brasileiros cresce exponencialmente entre jovens de 15 a 29 anos (Ministério da Saúde, 2018). A literatura especializada aponta que, justamente nesse período do desenvolvimento, existe uma tendência à exploração de experiências sexuais diversas, o que pode expor os jovens a experimentarem comportamentos de riscos à sua saúde (Alves \& Lopes, 2008; Arnett, 2000). Portanto, a realização de estudos empíricos junto a esse público torna-se fundamental, a fim de prevenir riscos, evitar consequências negativas para a saúde e bem-estar e promover saúde. A escolha pelo público feminino como foco do presente estudo 
deu-se a partir dos dados apresentados pela literatura, apontando que o sexo casual pode oferecer piores consequências à saúde das mulheres (Bersamin et al., 2014; Claxton \& van Dulmen, 2013; Fielder et al., 2014; Plummer et al., 2016).

Em vista do exposto, o objetivo deste estudo foi explorar e comparar a percepção de risco e os comportamentos de saúde, que envolvem o sexo casual, em universitárias com experiência de sexo casual (CEX) e sem experiência (SEX). Nesse sentido, entende-se comportamentos de saúde, como aqueles que têm o objetivo da manutenção e a proteção da própria saúde (Gaspar de Matos, 2012). Dessa maneira, espera-se contribuir para promover a saúde sexual, liberdade e satisfação no exercício da sexualidade das universitárias.

\section{Método}

\section{Delineamento}

Transversal comparativo entre grupos (CEX e SEX).

\section{Participantes}

Participaram deste estudo 1.133 universitárias de diferentes cursos e universidades do Brasil, que foram divididas em dois grupos: com experiência de sexo casual (CEX) $(n=804)$ e sem experiência de sexo casual (SEX) $(n=329)$. A média de idade do grupo CEX foi de 21,73 anos $(D P=2,17)$, e no grupo SEX foi de 21,05 anos $(D P=2,05)$. Os principais dados sociodemográficos da amostra estão especificados na Tabela 1 .

A amostra foi selecionada por conveniência entre aquelas mulheres que tiveram acesso à divulgação da pesquisa nas redes sociais e se propuseram a respondê-la. Os critérios de inclusão para participar neste estudo foram: ser mulher, ser brasileira, possuir entre 18 e 25 anos e ser estudante universitária de curso de graduação. Foram excluídas as mulheres que não haviam iniciado a vida sexual $(n=30)$, as que não eram brasileiras $(n=3)$ e não eram estudantes de cursos de graduação $(n=11)$. Utilizou-se o software Gpower para calcular o tamanho de efeito da amostra. Foi utilizado o teste estatístico para verificar as diferenças de médias entre dois grupos independentes, no $t$ test. $\mathrm{O}$ tamanho do encontrado para essa amostra é alto $(<0,80)$.

\section{Instrumentos}

1) Questionário de dados sociodemográficos e de saúde. Criado especialmente para este estudo, o questionário contém 15 questões de múltipla escolha, referentes aos dados sociodemográficos (escolaridade, idade, religião, estado civil, dados profissionais, entre outros) e 22 questões de múltipla escolha sobre comportamentos de saúde (frequência ao ginecologista, prática de exames preventivos e de detecção de infecções sexualmente transmissíveis, uso de métodos contraceptivos, histórico, hábitos e comportamento sexual). Algumas respostas não eram obrigatórias, tais como local de nascimento, ou de atual moradia, a fim de evitar constrangimento e receio de identificação dos dados pessoais.

2) Questionário sobre a percepção do sexo casual em universitárias. Questionário semiestruturado on-line, construído pelas autoras a partir dos dados da literatura disponível com o propósito de avaliar a percepção de riscos, comportamentos e crenças relacionadas ao sexo casual. O questionário foi composto por seis questões de múltipla escolha para investigar o uso consistente e inconsistente de preservativo em relações sexuais formais e casuais (e.g., uso de preservativo no sexo oral, anal e genital) e três questões apresentadas a partir de escalas do tipo Likert, que variavam de 0 (nenbum risco) a 10 (completo risco), para explorar as percepções de risco do sexo casual e percepção de risco para saúde do sexo, sem preservativo, com parceiro fixo e com parceiro casual (e.g., quanto acredita que o sexo casual envolve riscos). Quanto maior a pontuação, maior era a percepção de risco. Optou-se por criar um questionário específico para o presente estudo, uma vez que os instrumentos que avaliam comportamento sexual não são específicos em examinar essas condutas relacionadas ao sexo casual em mulheres. O questionário completo está anexado a este artigo, no apêndice 1.

\section{Procedimentos de Coleta de Dados e Éticos}

A pesquisa foi divulgada por meio de redes sociais (Whatsapp e Facebook) nas páginas do grupo de pesquisa e da Universidade onde as autoras estão inseridas e seus diversos compartilhamentos posteriores. Além disso, foi feita uma busca ativa por grupos de universitários e universidades nas redes sociais, além de divulgação junto a instituições universitárias e professores universitários, por e-mail e redes sociais. Foi direcionado aos grupos um link que continha o Termo 
Tabela 1.

Caracterização da Amostra

\begin{tabular}{|c|c|c|c|c|c|c|}
\hline \multirow{3}{*}{$\begin{array}{l}\text { Variáveis } \\
\text { Total de participantes }\end{array}$} & \multirow{2}{*}{\multicolumn{2}{|c|}{$\frac{\text { CEX }}{(n=804)}$}} & \multirow{2}{*}{\multicolumn{2}{|c|}{$\frac{\text { SEX }}{(n=329)}$}} & \multirow{2}{*}{\multicolumn{2}{|c|}{$\frac{\text { Total }}{(n=1133)}$}} \\
\hline & & & & & & \\
\hline & \multirow{2}{*}{$\begin{array}{l}\% \% \\
71\end{array}$} & \multirow{2}{*}{$\begin{array}{c}n \\
804 \\
\end{array}$} & \multirow{2}{*}{$\begin{array}{l}\% \\
29 \\
\end{array}$} & \multirow{2}{*}{$\begin{array}{c}\mathrm{n} \\
329\end{array}$} & \multirow{2}{*}{$\begin{array}{c}\% \\
100\end{array}$} & \multirow{2}{*}{$\begin{array}{c}n \\
1133 \\
\end{array}$} \\
\hline & & & & & & \\
\hline \multicolumn{7}{|l|}{ Área de Conhecimento* } \\
\hline Ciências Exatas e da Terra & 5,02 & 40 & 4,3 & 14 & 4,8 & 54 \\
\hline Ciências Biológicas & 3,6 & 29 & 4,9 & 16 & 4 & 45 \\
\hline Engenharias & 8,5 & 68 & 10,2 & 33 & 9 & 101 \\
\hline Ciências da Saúde & 14,1 & 112 & 14,8 & 48 & 14,3 & 160 \\
\hline Ciências Agrárias & 3,6 & 29 & 5,8 & 19 & 4,3 & 48 \\
\hline Ciências Sociais e Aplicadas & 30,2 & 240 & 26,5 & 86 & 29,1 & 326 \\
\hline Ciências Humanas & 22,5 & 179 & 21,8 & 71 & 22,3 & 250 \\
\hline Linguística, Letras e Artes & 8,9 & 71 & 8,6 & 28 & 8,8 & 99 \\
\hline Outros & 3,5 & 28 & 3,08 & 10 & 3,4 & 38 \\
\hline \multicolumn{7}{|l|}{ Religião das participantes** } \\
\hline Não tem religião & 39,8 & 320 & 30,4 & 100 & 25,5 & 420 \\
\hline Católica & 37,4 & 301 & 41,6 & 137 & 26,6 & 438 \\
\hline Evangélica & 8,7 & 70 & 12,8 & 42 & 6,8 & 112 \\
\hline Espírita & 11,6 & 93 & 9,7 & 32 & 7,6 & 125 \\
\hline Outras crenças & 8 & 65 & 9 & 30 & 5,8 & 95 \\
\hline Praticante desta religião & 35,7 & 287 & 52,3 & 172 & 27,8 & 459 \\
\hline \multicolumn{7}{|l|}{ Tipo de relacionamento* } \\
\hline Sem parceiro (a) fixo & 63 & 497 & 37 & 118 & 55 & 615 \\
\hline Com parceiro (a) fixo & 37 & 293 & 63 & 197 & 45 & 490 \\
\hline \multicolumn{7}{|l|}{ Responsabilidade financeira* } \\
\hline Independente & 22,4 & 161 & 13,7 & 40 & 19,9 & 201 \\
\hline Dependente & 77,6 & 557 & 86,3 & 252 & 80,1 & 809 \\
\hline
\end{tabular}

*O n total não corresponde ao total de participantes, pois não era uma resposta obrigatória.

** Poderiam marcar mais de uma resposta.

de Consentimento Livre e Esclarecido virtual e, posteriormente, os questionários, que foram respondidos e os dados foram armazenados na plataforma do Google Docs. O tempo médio estimado para responder a pesquisa foi de 45 minutos.

A presente pesquisa foi amparada pela Resolução 510/2016 do Conselho Nacional de Saúde, órgão do Ministério da Saúde que estipula as diretrizes e normas regulamentadoras de pesquisas com seres humanos. Além disso, foi submetida e aprovada pelo Comitê de Ética em Pesquisa da Universidade do Vale dos Sinos (por número CAAE).

\section{Análise de Dados}

Os dados obtidos pelo questionário on-line foram tabulados e analisados por meio do programa SPSS, 
versão 22.0. A comprovação dos critérios de supostos paramétricos, por meio do teste de Kolomogorov-Smirnov, evidenciou que os dados apresentam uma distribuição normal. Dessa forma, inicialmente foram realizadas análises descritivas dos dados. Os dados sociodemográficos e de saúde categóricos (histórico de saúde e experiências sexuais) foram analisados por meio de frequência, porcentagens e teste Qui-quadrado para a comparação entre os grupos CEX e SEX. Os dados numéricos (histórico de parceiros(as), experiências sexuais e dados clínicos e respostas do tipo Likert do questionário) foram analisados por meio das médias, desvio padrão e teste $t$ de Student, a fim de comparar as médias entre os grupos.

\section{Resultados}

A média de parceiros sexuais do grupo CEX foi de 2,58 $(D P=4,76)$ nos últimos seis meses, e a média das relações de sexo casual foi de 5,59 $(D P=14,9)$ no mesmo período. Na Tabela 2 estão apresentados outros dados sobre o histórico da vida sexual das participantes. Observa-se que as mulheres CEX iniciaram a vida sexual significativamente mais cedo que as do grupo SEX. No grupo CEX, a média de idade de início da vida sexual das participantes foi de 16,33 anos $(D P=$ $2,60)$, enquanto, no grupo SEX, a idade média de início da vida sexual foi de 17,20 anos $(D P=2,36)$, demonstrando uma diferença significativa entre as médias de idades $(t=5,07 ; p<0,001)$.

Em relação aos comportamentos de saúde, que são aqueles que tem como objetivo a manutenção e a proteção da sua saúde, o grupo CEX em geral demonstrou ter maiores cuidados com a saúde sexual, especialmente em relação à frequência de ida ao ginecologista, realização de exames preventivos ginecológicos, uso de preservativo, investigação e tratamento de ISTs, de maneira significativa, conforme Tabela 3 . As exceções foram a frequência de ida ao médico para revisões e o uso de pílula anticoncepcional, que foi semelhante entre os grupos.

Ao comparar as médias da percepção de riscos em relação ao uso de preservativo, na Tabela 4 , chama a atenção que, em ambos os grupos, as médias de percepção de risco são muito maiores quando o parceiro sexual é casual, do que formal. Na comparação geral da média da percepção de risco do sexo desprotegido com parceiro(a) fixo $(M=5,52 \mathrm{DP}=2,75)$ e da média geral da percepção de risco do sexo desprotegido com parceiro(a) casual $(M=9,18 D P=1,52)$, houve diferença significativa entre essas médias $(t=4,30 ; p=$ $0,004)$. Ficou evidente a diferença significativa de percepção, na qual o grupo SEX percebe maiores riscos do sexo casual, e parceiros casuais do que o grupo CEX.

Ao comparar o uso consistente de preservativo na relação sexual, houve uso mais consistente entre as universitárias na prática do sexo casual, em relação as universitárias que praticam o sexo apenas na relação formal. Ambos os grupos demonstraram ter o maior desuso de preservativo no sexo oral. A diferença mais significativa é relativa à prática do sexo genital, em que a taxa do uso de preservativo usado na relação casual cai para 51,6\% quando a o sexo é dentro da relação formal. O detalhamento dos dados está apresentado na Tabela 5.

\section{Discussão}

Em vista do exposto, o objetivo deste estudo foi explorar e comparar a percepção dos riscos à saúde física e a adesão a comportamentos de saúde relacionados ao sexo casual, em universitárias com e sem experiência de sexo casual. De maneira geral, foi possível observar que existe diferença significativa entre os grupos em relação à percepção de risco e comportamentos de saúde. O grupo CEX apresentou mais comportamentos de

Tabela 2.

Teste Qui-Quadrado e Nivel de Significancia dos Dados de Histórico Sexual das Universitárias dos Grupos CEX e SEX

\begin{tabular}{|c|c|c|c|c|c|c|}
\hline \multirow{2}{*}{ Histórico de comportamento sexual } & \multicolumn{2}{|c|}{$\mathrm{CEX}(n=804)$} & \multicolumn{2}{|c|}{$\operatorname{SEX}(n=329)$} & \multirow{2}{*}{$\mathrm{X}^{2}$} & \multirow{2}{*}{$g l$} \\
\hline & $\%$ & $N$ & $\%$ & $N$ & & \\
\hline Somente com homens & 68,7 & 552 & 90,3 & 297 & $70,683^{* *}$ & 2 \\
\hline Somente com mulheres & 1,6 & 13 & 3 & 10 & & \\
\hline Com homens e mulheres & 29,7 & 239 & 6,7 & 22 & & \\
\hline
\end{tabular}

$* p<0,005 * * p<0,001$. 
Tabela 3.

Frequência e Comparativo de Comportamentos de Saúde entre os Grupos

\begin{tabular}{|c|c|c|c|c|c|c|}
\hline \multirow{2}{*}{ Variáveis } & \multicolumn{2}{|c|}{$\operatorname{CEX}(n=804)$} & \multicolumn{2}{|c|}{$\operatorname{SEX}(n=329)$} & \multirow{2}{*}{$x^{2}$} & \multirow{2}{*}{$g l$} \\
\hline & $\%$ & $n$ & $\%$ & $n$ & & \\
\hline \multicolumn{7}{|l|}{ Frequência ao ginecologista } \\
\hline Não costuma ir & 19,2 & 154 & 23,7 & 78 & & \\
\hline 1 vez ao ano & 70,6 & 568 & 65,7 & 216 & 3,293 & 2 \\
\hline A cada 2 anos ou mais & 10,2 & 82 & 10,6 & 35 & & \\
\hline \multicolumn{7}{|c|}{ Exames preventivos ginecológicos } \\
\hline Não costuma fazer & 22 & 177 & 32,2 & 107 & $14,117 *$ & \\
\hline 1 vez ao ano & 66,5 & 535 & 56,5 & 186 & & 2 \\
\hline A cada 2 anos ou mais & 11,5 & 92 & 10,9 & 36 & & \\
\hline Uso método contraceptivo & 91,2 & 733 & 83,3 & 274 & $14,691 *$ & 1 \\
\hline Pílula anticoncepcional & 56,1 & 451 & 60,4 & 198 & 1,737 & 1 \\
\hline Preservativo & 62,9 & 506 & 54,7 & 163 & $6,185^{*}$ & 1 \\
\hline Exames detecção ISTs & 70,8 & 569 & 53,8 & 177 & $29,901 * *$ & 1 \\
\hline Tratamento ISTs/DSTs & 54,9 & 146 & 39,8 & 33 & $5,795 *$ & 1 \\
\hline
\end{tabular}

$* p<0,005 * * p<0,001$

saúde e maiores cuidados em relação à saúde sexual que o grupo SEX. Ambos os grupos perceberam o sexo casual desprotegido com maior risco do que o sexo desprotegido dentro de relações formais. O grupo SEX percebeu maior risco do sexo casual sem preservativo e maior uso inconsistente de preservativo em relação ao grupo CEX.

Os dados examinados demonstraram que, em ambos os grupos, foi menor a percepção de risco do sexo desprotegido nos relacionamentos formais se comparados aos casuais. Ao passo que as relações se tornam estáveis, o uso do preservativo passa a ser inconsistente, o que já é referido pela literatura (Alves \& Lopes, 2008; Harvey et al., 2007). A partir de então, o risco de transmissão de infecções sexualmente transmissíveis pode aumentar. Esses achados contrariam estudos (Bersamin et al., 2014; Fielder et al., 2014; Lyons, 2016) que colocam o sexo casual como um fator de risco para a saúde física de adultos emergentes. Pode-se perceber, nesses estudos, que existe uma grande tendência a atrelar o sexo casual ao risco de contrair ISTs, enquanto este estudo demonstrou que o uso consistente de preservativo é maior pelas universitárias que praticam o sexo casual do que aquelas que praticam sexo apenas dentro de relações formais.

O sexo casual foi percebido pelas universitárias, de ambos os grupos, com um alto risco para a saúde. Nesse sentido, os modelos de percepção de risco em saúde (Leventhal et al., 2016; Slovic, 2000) auxiliam a compreender as diferenças encontradas. O risco real de contrair uma IST na relação sexual sem preservativo é alto, seja numa relação formal ou casual. Porém, ao perceber maior risco no sexo casual, o uso do preservativo tende a ser adotado como medida de prevenção para possíveis infecções, doenças ou gravidez indesejada. Estar em uma relação estável não previne a mulher do risco de contrair doenças. Portanto, o uso do preservativo mostra-se como uma das maneiras mais eficazes para a prevenção de grande parte das consequências negativas, tais como gravidez indesejada, aborto e transmissão de ISTs (OMS, 2013; Dourado et. al., 2015). A percepção de risco mais ajustada das universitárias pode contribuir com a criação de um repertório de comportamentos em saúde funcionais e adaptativos, culminando na adoção de condutas que visem a manutenção e a proteção da sua saúde (Gaspar de Matos, 2012). A partir desta 
Tabela 4.

Comparação de Médias da Percep̧ãa de Risco em Relação ao Parceiro entre os Grupos CEX e SEX

\begin{tabular}{|c|c|c|c|c|c|c|c|}
\hline \multirow{2}{*}{ Percepção de risco } & \multirow{2}{*}{ Mínimo } & \multirow{2}{*}{ Máximo } & \multicolumn{2}{|c|}{$\operatorname{CEX}(n=804)$} & \multicolumn{2}{|c|}{$\operatorname{SEX}(n=329)$} & \multirow{2}{*}{$\mathrm{t}$} \\
\hline & & & $M$ & $D P$ & $M$ & $D P$ & \\
\hline Sexo casual & 0 & 10 & 7,01 & 2,39 & 7,84 & 2 & $-5,816^{* *}$ \\
\hline $\begin{array}{l}\text { Sexo com parceiro (a) fixo sem } \\
\text { preservativo }\end{array}$ & 0 & 10 & 5,69 & 2,77 & 5,35 & 2,73 & 1,895 \\
\hline $\begin{array}{l}\text { Sexo com parceiro (a) casual sem } \\
\text { preservativo }\end{array}$ & 0 & 10 & 9 & 1,76 & 9,36 & 1,28 & $-3,825^{* *}$ \\
\hline
\end{tabular}

${ }^{* *} p<0,001$

Tabela 5.

Comparativo do Uso de Preservativo na Prática Sexual no Contexto de Relação Casual e Formal

\begin{tabular}{|c|c|c|c|c|c|c|c|c|}
\hline & & \multirow{2}{*}{\multicolumn{2}{|c|}{$\begin{array}{l}\text { CEX }(n=804) \\
\text { Relação casual } \\
\end{array}$}} & \multirow{2}{*}{\multicolumn{2}{|c|}{$\begin{array}{l}\text { SEX }(n=329) \\
\text { Relação formal }\end{array}$}} & \multirow{3}{*}{$p$} & \multirow{3}{*}{$x^{2}$} & \multirow{3}{*}{$G L$} \\
\hline & & & & & & & & \\
\hline & & $\%$ & $n$ & $\%$ & $n$ & & & \\
\hline \multirow[t]{3}{*}{ Sexo Oral } & Uso não consistente & 86,6 & 696 & 89,1 & 293 & 0,2532 & 1,304 & 1 \\
\hline & Uso consistente & 13,4 & 108 & 10,9 & 36 & & & \\
\hline & Total & 100 & 804 & 100 & 329 & & & \\
\hline \multirow[t]{3}{*}{ Sexo Anal } & Uso não consistente & 29,2 & 235 & 59,9 & 197 & 0,001 & 92,95 & 1 \\
\hline & Uso consistente & 70,8 & 569 & 40,1 & 132 & & & \\
\hline & Total & 100 & 804 & 100 & 329 & & & \\
\hline \multirow[t]{3}{*}{ Sexo Genital } & Uso não consistente & 26,1 & 210 & 61,8 & 203 & 0,001 & 127,5 & 1 \\
\hline & Uso consistente & 73,9 & 594 & 38,2 & 126 & & & \\
\hline & Total & 100 & 804 & 100 & 329 & & & \\
\hline
\end{tabular}

discussão, abre-se espaço para transformar a associação entre sexo casual e risco para uma associação entre sexo sem preservativo e risco.

Os relacionamentos fixos, geralmente, estão associados a maior confiança entre os parceiros, o que leva ao enfraquecimento de crenças de vulnerabilidade em relação a transmissão de infecções pelo parceiro (Harvey et al., 2007). Maior confiança, por sua vez, leva a diminuir as intenções de uso do preservativo. Porém, a confiança não exclui a possibilidade do(a) parceiro(a) de estar contaminad (a) com alguma IST. Além disso, deixar de ter relações sexuais sem preservativo pela confiança nos exames laboratoriais para detecção de doenças pode ser arriscado. A contaminação por ISTs apresenta uma janela imunológica, que pode gerar resultados falsos negativos (Organização Mundial da Saúde, 2013).
Além disso, pode haver contaminação devido a relações sexuais com parceiros extra relacionamento sem preservativo. Dados demonstram que apenas 46,3\% das pessoas que têm, ao mesmo tempo, relações estáveis e casuais, usam preservativo (Berquó et al., 2008). Então, o fato de estar em uma relação fixa não exclui a possibilidade de contaminação, por consequência, requer o uso de preservativo (Berquó et al., 2008). Nesse sentido, a baixa percepção de risco, do sexo sem preservativo no relacionamento formal, mostra-se um verdadeiro risco para a saúde da mulher.

Pode-se perceber que o grupo CEX possui maior diversidade de experiência com pessoas do mesmo sexo e iniciaram mais cedo a vida sexual em relação ao SEX. Além disso, o grupo CEX possui a média de parceiros maior que o grupo SEX (CEX $M=3$, nos últimos 6 
meses). Porém, contrariando os dados de estudos anteriores, (Bersamin et al., 2014; Fielder \& Carey, 2010; Lyons, 2016), as universitárias com maior experiência sexual são as que apresentaram melhores comportamentos de manutenção e da proteção da sua saúde. O grupo CEX demonstrou maior frequência ao ginecologista, realização de exames preventivos ginecológicos, uso de preservativo, investigação e tratamento de ISTs se comparado ao grupo SEX.

Os dados encontrados sugerem que o grupo SEX foi quem apresentou menor cuidado e maiores comportamentos de risco à saúde, pois usam menos preservativo e logo estão mais expostas aos riscos reais. Isso contraria um estudo recente sobre sexo casual e diagnóstico de ISTs, realizado com uma amostra norte-americana de adultos emergentes. Os comportamentos de risco avaliados foram: ter experiência de sexo casual, ter tido três ou mais parceiros casuais e iniciar a vida sexual antes dos 18 anos (Lyons, 2016). Faz-se necessário, portanto, discutir de maneira ampla, e sem preconceitos, o que de fato representa risco, pois, no presente estudo, ele não está relacionado à diversidade de parceiros e experiências, e sim à baixa percepção de risco e ao não uso de preservativo.

Outro fator que chamou a atenção está relacionado ao uso inconsistente de preservativo na prática do sexo oral, por ambos os grupos. Esse é um dado preocupante, que também pode estar atrelado à falta de informação e a baixa percepção de risco em relação a essa prática. Conforme estudo realizado (Chambers, 2007) sobre as percepções do sexo oral na população universitária, muitos jovens desconheciam riscos e informações sobre transmissão e meio de proteção contra ISTs. Além disso, as mulheres eram as que menos se protegiam ao receber sexo oral, aumentando o seu risco de contrair infecções. O sexo oral é a porta de entrada de vírus, tais como o herpes labial e o HPV, que podem trazer consequências negativas para a saúde da mulher. No caso do HPV, as consequências da transmissão podem variar desde infecção de colo de útero ao desenvolvimento de câncer de colo do útero, vulva, vagina, laringe, faringe, boca (Petito et al., 2015; Plummer et al., 2016). Além destas, outras ISTs também podem ser transmitidas pelo sexo oral. Para tanto, esses achados demonstram um grande desafio no desenvolvimento de promoção e prevenção em saúde sexual, no sentido de informar sobre os reais riscos envolvidos na prática de sexo oral. Esse desafio pode se estender à indústria, no sentido de desenvolver meios de proteção que possam ter maior aderência do público feminino.

\section{Considerações Finais}

Os achados deste estudo nos permitem repensar as associações entre o sexo casual e os riscos à saúde. As percepções de risco atreladas às práticas e a comportamentos sexuais podem levar ao aumento de uso de proteção, comportamentos de saúde e autocuidado. À medida que o sexo casual é percebido com maior risco, em relação ao sexo dentro de relações formais, é possível que medidas de proteção sejam adotadas com maior frequência.

Este estudo sugere uma série de desafios para pais, educadores, psicólogos e demais profissionais da saúde: promover informação e esforços para a prevenção de transmissão de infecções sexualmente transmissíveis. A educação e estímulo da agência sexual demonstra ser uma via para alcançar tais objetivos. Adequar as distorções e percepções dos riscos, envolvidos na prática sexual, também pode auxiliar a mulher para a tomada de decisões que possam aliar saúde e satisfação.

As limitações deste estudo compreendem o fato de a amostra ter sido selecionada por conveniência por meio das redes sociais, o que fragiliza por um lado por não permitir a generalização dos achados. Porém, permitiu um grande alcance de participantes e a garantia de que as universitárias pudessem responder a pesquisa sobre um tema de difícil abordagem, com a certeza do anonimato. Além disso, o delineamento transversal não permite identificar relações de causa-efeito das percepções de risco e condutas frente ao sexo. O tema sexualidade ainda é revestido de tabus e complexidade na sua discussão. Nesse sentido, mesmo com anonimato, questões referentes à desejabilidade social podem ter interferido nas respostas das universitárias, tanto no sentido de omitir informações, quanto a de exagerar em relação à expressão do exercício da sexualidade.

\section{Referências}

Abbey, A., Saenz, C., \& Buck, P. O. (2005). The cumulative effects of acute alcohol consumption, individual differences and situational perceptions on sexual decision-making. Journal of Studies on Alcohol, 66, 82-90. doi: 10.15288/jsa.2005.66.82

Alves, A. S., \& Lopes, M. H. M. B. (2008). Conhecimento, atitude e prática do uso de pílula e preservativo entre adolescentes universitários. Revista Brasileira de Enfermagem, 61(1), 11-17. doi: 10.1590/S0034-71672008000200005

Psico-USF, Bragança Paulista, v. 26, n. 1, p. 117-128, jan./mar. 2021 
Arnett, J. J. (2000). Emerging adulthood: A theory of development from the late teens through the twenties. American Psychologist, 55(5), 469-480. doi: 10.1037/0003-066X.55.5.469

Berquó, E., Barbosa, R. M., \& Lima, L. P. De. (2008). Trends in condom use: Brazil 1998 and 2005. Revista de Saúde Pública, 42 Suppl 1, 34-44. doi: S0034-89102008000800006

Bersamin, M.M., Zamboanga, B. L., Schwartz, S. J., Brent Donnellan, M., Hudson, M., Weisskirch, R. S., ... Jean Caraway, S. (2014). Risky business: Is there an association between casual sex and mental health among emerging adults? Journal of Sex Research, 51(1), 43-51. doi: 10.1080/00224499.2013.772088

Campbell, A. (2008). The morning after the night before: Affective reactions to one-night stands among mated and unmated women and men. Human Nature, 19(2), 157-173. doi: 10.1007/s12110-008-9036-2

Chambers, W. C. (2007). Oral Sex: Varied behaviors and perceptions in a college population. The Journal of Sex Research, 44(1), 28-42. doi: 10.1080/00224490709336790

Claxton, S. E., \& van Dulmen, M. H. M. (2013). Casual sexual relationships and experiences in emerging adulthood. Emerging Adulthood, 1(2), 138-150. doi: $10.1177 / 2167696813487181$

Dourado, I., MacCarthy, S., Reddy, M., Calazans, G., \& Gruskin, S. (2015). Revisiting the use of condoms in Brazil. Revista Brasileira de Epidemiologia, 18(1), 6388. doi: 10.1590/1809-4503201500050006

Fielder, R. L., \& Carey, M. P. (2010). Predictors and consequences of sexual "hookups" among college students: A short-term prospective study. Archives of Sexual Behavior, 39(5), 1105-1119. doi: 10.1007/ s10508-008-9448-4

Fielder, R. L., Walsh, J. L., Carey, K. B., \& Carey, M. P. (2014). Sexual hookups and adverse health outcomes: A longitudinal study of first-year college women. Journal of Sex Research, 51(2), 131-144. doi: 10.1080/00224499.2013.848255

Gaspar de Matos, M. (2012). Psicologia da Saúde, saúde pública e saúde internacional. Análise Psicológica, 22(3), 449-462. https://doi.org/10.14417/ap.215

Gilchrist, H., Smith, K., Magee, C. A., \& Jones, S. (2012). A hangover and a one-night stand: Alcohol and risky sexual behaviour among female students at an Australian University. Youth Studies Australia, 31(2), 35-43.

Harvey, S. M., Beckman, L. J., Gerend, M. A., Bird, S. T., Huszti, H. C., Galavotti, C., ... Huszti, H. C. (2007). A conceptual model of women's condom use intentions: Integrating intrapersonal and relationship factors. AIDS Care, 18(7), 698-709. doi:10.1080/13548500500294435

Hoyle, R. H., Fejfar, M. C., \& Miller, J. D. (2000). Personality and sexual risk taking: A quantitative review. Journal of Personality, 68(6), 1203-1231. doi: 10.1111/1467-6494.00132

Leventhal, H., Phillips, L. A., \& Burns, E. (2016). Modelling management of chronic illness in everyday life: A Common-Sense Approach. Psychological Topics, 25(1), 1-18.

Lyons, H. A. (2016). Heterosexual casual sex and STI diagnosis: A Latent Class Analysis. In ternational Journal of Sexual Health, 76(11). doi: 10.1080/19317611.2016.1210711

Mendão, M. A. C., \& Biscaia, C. (2015). Perceção de risco no one-night stand - Um estudo com adultos emergentes (Dissertação de mestrado). Universidade de Évora.

Ministério da Saúde. (2018). Painel de Indicadores Epidemiológicos. Departamento de Vigilância, Prevenção e Controle das IST, HIV/Aids e Hepatites Virais. Recuperado de www.aids.gov.br/pt-br/ gestores/painel-de-indicadores-epidemiologicos

Organização Mundial da Saúde. (2013). Diagnóstico laboratorial de doenças sexualmente transmissíveis, incluindo o vírus da imunodeficiência humana. World Health Organization, 175-188. Recuperado de www.who.int/reproductivehealth

Petito, G., Oliveira Júnior, S. M., Petito, A. D. C., \& Saddi, V. A. (2015). Human papillomavirus in head and neck carcinomas: Prevalence and clinicopathological relationship. Jornal Brasileiro de Doenças Sexualmente Transmissiveis, 27(1-2), 6-8. doi: 10.5533/DST-2177-8264-2015271-202

Plummer, M., de Martel, C., Vignat, J., Ferlay, J., Bray, F., \& Franceschi, S. (2016). Global burden of cancers attributable to infections in 2012: A synthetic analysis. The Lancet Global Health, 4(9), e609-e616. doi: 10.1016/S2214-109X(16)30143-7

Slovic, P. (2000). The perception of risk. Recuperado de https://books.google.com.br/books?hl=pt-BR\&l 
$\mathrm{r}=\& \mathrm{id}=\mathrm{BLYyDQAAQBAJ \& oi}=$ fnd $\& \mathrm{pg}=\mathrm{PT} 8 \& \mathrm{~d}$ $\mathrm{q}=$ Perception + of + Risk\&ots $=\mathrm{pZU}$ Zuw PmGmT\&s ig $=$ Mw0o7f-hH4BD-5E3ftapnbKW8rQ\# $\#_{\mathrm{v}}=$ one page\&q $=$ Perception of Risk\&f $=$ false

Vrangalova, Z. (2015). Does casual sex harm college students' well-being? A longitudinal investigation of the role of motivation. Archives of Sexual Behavior, 44(4), 945-959.doi: 10.1007/s10508-013-0255-1

Wentland, J. J., \& Reissing, E. D. (2011). Taking casual sex not too casually: Exploring definitions of casual sexual relationships. The Canadian Journal of Human Sexuality, 20(3),
75-91. Recuperado de sexresearchandthecity.com/ wp-content/uploads/2011/11/Taking-casual-sex-not-too-casually.pdf

Wentland, J. J., \& Reissing, E. (2014). Casual sexual relationships: Identifying definitions for one night stands, booty calls, fuck buddies, and friends with benefits. The Canadian Journal of Human Sexuality, 23(3), 167-177. https://doi.org/10.3138/cjhs.2744

Recebido em: 10/09/2018

Reformulado em: 09/01/2020

Aprovado em: 18/03/2020 


\section{Apêndice 1}

Questionário sobre a percep̧cão do sexo casual em universitárias*

Marque o quanto você acredita que o sexo casual envolva riscos, considerando '0' para nenhum risco e '10' para muitos riscos:

$$
\text { (1)-(2)-(4)-(5)-(10) }
$$

Marque a opção sobre o seu hábito de uso do preservativo no SEXO CASUAL (Pode marcar mais de uma opção):

Uso preservativo no sexo genital

Marque a opção sobre o seu hábito de uso do uso preservativo no RELACIONAMENTO FORMAL (Pode marcar mais de uma opção):

Uso preservativo no sexo oral

Marque, em uma escala de 0 a 10, qual o grau de risco para a sua saúde de fazer sexo com um PARCEIRO FIXO sem preservativo:

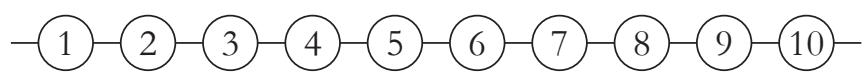

Marque, em uma escala de 0 a 10, qual o grau de risco para a sua saúde de fazer sexo com um PARCEIRO CASUAL sem preservativo:

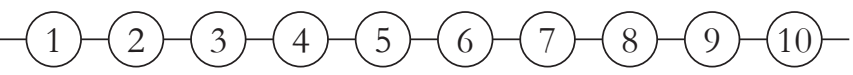

* Instrumento elaborado pelos autores.

Uso preservativo no sexo oral


Sobre os autores:

Andresa Pinho Soster é Psicóloga, Pós-Graduada em Gestão e Desenvolvimento de Pessoas, Pós-Graduada em Psicoterapia Cognitivo-Comportamental, Mestre em Psicologia Clínica. Atualmente doutoranda em Sexualidade Humana, pela Universidade do Porto, Portugal. Membro WAS (World Association for Sexual Health) e da FBTC (Federação Brasileira de Terapias Cognitivas). Psicóloga Clínica, Professora, e Supervisora Clínica em Terapia Cognitiva-Comportamental. Pesquisadora na área de sexualidade, gênero feminino e sexo casual.

ORCID: https://orcid.org/0000-0001-6996-3778

E-mail: apsoster@gmail.com

Miguel Luis Alves de Souza é Acadêmico do Curso de Psicologia na Universidade do Vale do Rio dos Sinos (UNISINOS) e bolsista de iniciação científica do Conselho Nacional de Desenvolvimento Científico e Tecnológico (CNPq). ORCID: https://orcid.org/0000-0002-9012-9996

E-mail:mlalvesdesouza@outlook.com

Elisa Kern de Castro é Psicóloga, Doutora em Psicologia Clínica e da Saúde e professora adjunta do Programa de Pós Graduação em Psicologia Clínica da Universidade do Vale do Rio dos Sinos (UNISINOS).

ORCID: https://orcid.org/0000-0002-1290-7561

E-mail: elisa.kerndecastro@gmail.com

Contato com os autores:

Rua Major Demétrius, 206, Bairro Salgado Filho

Gravataí-RS, Brasil

CEP: 94020-390 